\title{
Hydrodynamic forces acting on vertically translating bodies in free surface water
}

\author{
F. Kerger $^{1,2}$, S. Detrembleur ${ }^{1}$, P. Archambeau ${ }^{1}$, S. Erpicum ${ }^{1}$, \\ B. J. Dewals ${ }^{1,2}$ \& M. Pirotton ${ }^{1}$ \\ ${ }^{1}$ Laboratory of Hydrology, \\ Applied Hydrodynamics and Hydraulic Constructions, \\ Institute of Civil Engineering, ArGEnCO Department, Liège University, \\ Liège, Belgium \\ ${ }^{2}$ Belgian Fund for Scientific Research F.R.S-FNRS, Belgium
}

\begin{abstract}
The dynamics of free surface flow induced by a partially or totally submerged moving body generates non-straightforward effort distribution. In the design process of moving hydraulic structures and their operation device, the pressure field acting on them is of prime interest. A fundamental example of such a phenomenon, namely the vertical uplift of a submerged horizontal rigid sheet, is hereafter experimentally analyzed. The present study includes the description of the experimental apparatus built in the $\mathrm{HACH}$ laboratory and the description of the rigorous experimental procedure used. The purpose of the analysis is to identify the fundamental hydrodynamic mechanisms involved.
\end{abstract}

Keywords: flap gate, experimental hydrodynamics, fluid structure interaction.

\section{Introduction}

The dynamics of free surface flow induced by the essentially vertical motion of a submerged body is a complex phenomenon. In particular, it remains challenging to predict the pressure distribution generated by the flow on the body. Phenomenon intricacy results from the underlying relationship between the pressure field surrounding the moving body and the velocity field resulting from its movement. Indeed, even if the pressure field arises from the flow, one must conceive of this field as the driving force of the flow. From a civil engineering point of view, several hydraulic structures may be assigned to this category of 
phenomena. For instance, flap gate movement, sealing the lock by coming from a chamber at the bottom of the lock foundation raft, is an evocative example.

To the author's knowledge, neither general theory nor design method has been proposed in literature. Traditionally, the design of this kind of structure is performed by creating an instrumented physical scale model in the laboratory. Such an approach has been lead for the flap gate motion by Canor [1]. He concluded that many phenomena appearing during the uplifting of the gate are still poorly understood. Sometimes, design table, expressed in terms of nondimensional parameters and derived from instrumented scale model, are available for a few practical applications. See Wiggert and Schmausser [2] for example of such tables. In the last decades, computational fluid dynamics has been providing an approach of increasing efficiency to design hydraulic structures. Used on its own or combined with a physical model, numerical simulation allows designers to avoid extensive and expensive optimization on a scale model, as shown by Dewals et al. [3]. However, developing such software is computationally difficult because it is needed to solve the incompressible Navier-Stokes equations in the vertical plane and track the free surface in parallel. In particular, such a step requires a good understanding of the phenomena involved in the simulated application. Detrembleur et al. [4] introduces a method based on an original finite volume scheme on structured grids and a Level Set Method for tracking the free surface.

Therefore, authors propose in this study to focus on a more fundamental and theoretical case, namely the vertical uplifting of a metallic rigid sheet from the pool bottom up to the free surface of the still water. This simplification leads to a better identification of the basic phenomena arising during the translation. This paper is organized as follows. Section 2 details the apparatus and the procedure of the experiments performed at the $\mathrm{HACH}$ laboratory. Section 3 proposes a dimensional analysis of the problem. Section 4 analyses the results of the campaign and a new model for interpretation.

\section{Original experiments}

\subsection{Experimental apparatus}

The experimental apparatus is composed of a $10 \mathrm{~m}$ by $2 \mathrm{~m}$ pool in sealed masonry based on a concrete slab. Visualisation of the flow is efficient through a Plexiglas wall, which replaces the masonry in the central part of the pool (Figure 1a). The rigid horizontal sheet consists of an aluminium orthotropic structure stuck between two Plexiglas panels (Figure 1b). During the experiments, hollows in the plate are filled with water and allow us to incorporate pressure gauges. The unit is $2 \mathrm{~m}$ long, $0.76 \mathrm{~m}$ wide and $0.16 \mathrm{~m}$ thick. The submerged weight is $64 \mathrm{~kg}$. Moving the rigid sheet is achieved by a system of ropes and pulleys (Figure 1c).

Two operation devices have been used during the experimental campaign. The first one is an adjustable counterweight, which has the major advantage to allow a highly repeatability of trials and to appraise the overall power provided to the sheet. The second one is a crank, which moves the panel at a constant speed. 


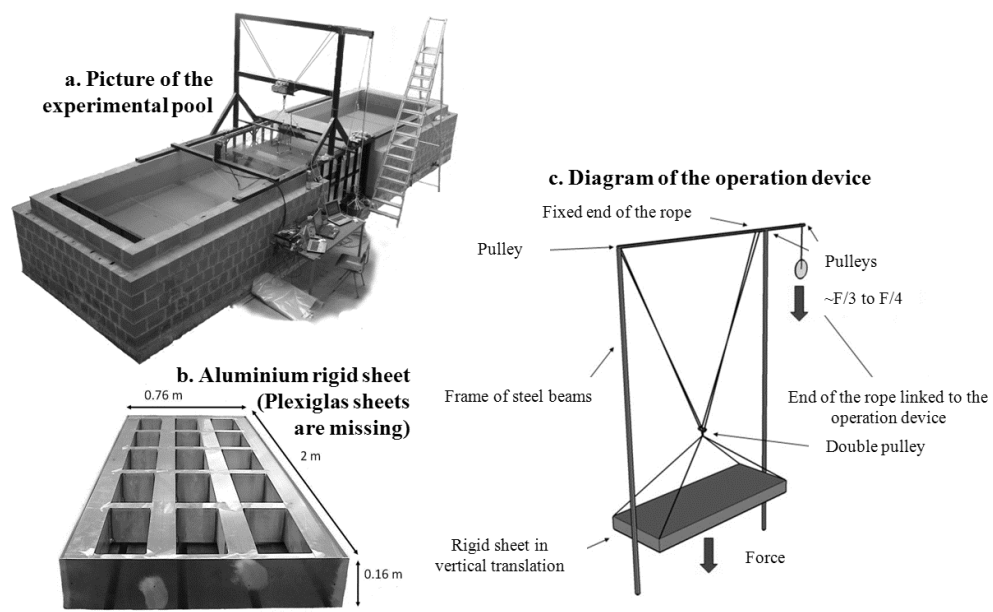

Figure 1: Experimental apparatus.

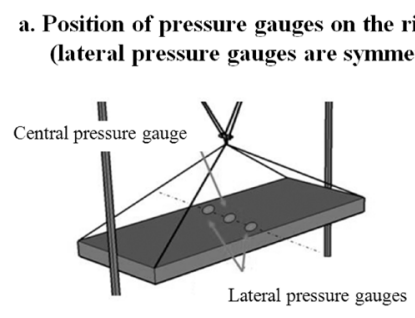

Figure 2: Measurement system.

\subsection{Measurement system}

The measurement system includes at first five pressure gauges (KELLER series 41 capacitive transmitters). Manufacturer ensures an accuracy of $\pm 1 \mathrm{~mm} \mathrm{H}_{2} \mathrm{O}$. On the one hand, three of these pressure gauges are fastened onto the central line of the horizontal panel, in parallel to its small side (Figure 2a). They are alternatively oriented towards the upper side and the lower side of the plate. On the other hand, two of them are held on $20 \mathrm{~mm}$ below the free surface and exactly above the two former ones. They provide the temporal evolution of the free surface elevation. In addition, the exact position of the rigid sheet is measured by using a potentiometer (Rheostats Bourns 3590S). The potentiometer is fastened to the shaft of a pulley (Figure 2b). 


\subsection{Experimental campaign}

The overall experimental campaign carried out within the Laboratory of Applied Hydrodynamics and Hydraulic Constructions $(\mathrm{HACH})$ of the University of Liege involved the following trials:

- two sets of 15 identical trials in which the rigid sheet motion is controlled by an adjustable counterweight ( $20 \mathrm{~kg}$ and $30 \mathrm{~kg})$;

- one set of 25 trials in which the rigid sheet velocity is kept constant along the trial (various steady velocities from $80 \mathrm{~mm} / \mathrm{s}$ to $105 \mathrm{~mm} / \mathrm{s}$ ).

\subsection{Experimental procedure}

The operation device is triggered off by discharging the counterweight hanged aloft or by rotating the crank. As a result, the rigid sheet, kept horizontal, moves from the bottom of the pool up to the free surface. During the translation, the measurement system records the temporal evolution of the pressure at a central and lateral point of the sheet on its upper and lower sides, the free-surface elevation directly above the central and lateral pressure gauge, and the position (depth) of the rigid sheet. Collected primary data are then processed in order to give average values of the parameters for several similar trials and determine the value associated to the confidence interval. The Confidence Interval (IC) chosen in this paper is $95 \%$. All charts of the present paper are thus given with the correspondent IC.

Subsequently, statistically processed measured pressures are broken down into several contributions so that all the relevant information may be extracted from the measured data (Figure 3). By this process, the so-called drag pressure is defined as the hydrodynamic pressure resulting from the fluid motion augmented by the free surface variation above the rigid sheet (for pressures measured on the lower side of the sheet, this last contribution is clearly zero). Physically, it consists of excluding the hydrostatic component from the measured pressure and keeping only the hydrodynamic component. Authors point out here the fundamental feature of this parameter: characterizing the behaviour and identifying factors influencing the drag pressure is the core of the present study.

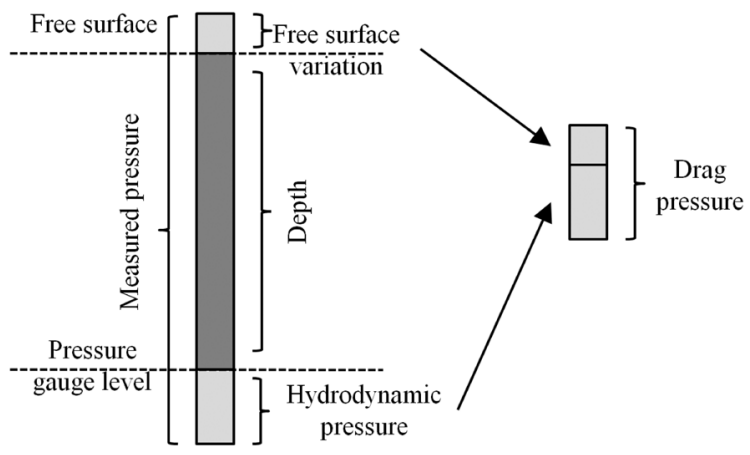

Figure 3: Definition of the drag pressure. 


\section{Dimensional analysis}

The pressure coefficient for a moving body in air (aerodynamics) is a traditional result given for instance by Candel [6]:

$$
C_{p}[-]=\frac{p}{\rho v^{2} / 2}=\mathrm{f}\left(\operatorname{Re}_{s}, \frac{x}{l}\right)
$$

Three mechanisms account for the distribution of $\mathrm{C}_{\mathrm{p}}$. First, part of the pressure field around the body originates from the pressure decrease induced by the increase of the fluid velocity module. This mechanism is well described by the Bernoulli equation $p=0.5 \rho v^{2}$. Second, a negative pressure appears behind the rigid sheet in translation due to the flow separation at the sharp ends of the panel. Third, the natural viscosity of water has a slight contribution to the creation of drag pressure around a moving body.

Application of the Vaschy-Buckingham theorem on the current experiment (see Kerger [5]) leads to the identification of the Pressure Coefficient $C_{p}$ for the upper face

$$
C_{p}[-]=\frac{p}{\rho v^{2} / 2}=\mathrm{f}\left(\mathrm{Fr}_{s}, \operatorname{Re}_{s}, \frac{a}{g}, \frac{x}{l}, \frac{h_{s}}{l}\right)
$$

Where Fr [-] $=\mathrm{v}_{\mathrm{s}} /\left(\mathrm{gh}_{\mathrm{s}}\right)^{1 / 2}$ is the sheet Froude number, Re [-] $=\rho \mathrm{v}_{\mathrm{s}} \mathrm{l} / \mu$ is the sheet Reynolds number, a $\left[\mathrm{ms}^{-2}\right]$ is the sheet acceleration, $\mathrm{g}\left[\mathrm{ms}^{-2}\right]$ is the gravity acceleration, $x[\mathrm{~m}]$ is the position on the sheet, $1[\mathrm{~m}]$ is the sheet width, $\mathrm{v}_{\mathrm{s}}\left[\mathrm{ms}^{-1}\right]$ is the sheet velocity, $h_{s}[\mathrm{~m}]$ is the sheet depth for the upper face or the length between the sheet and the bottom of pool for the lower face, $\rho\left[\mathrm{kg} \mathrm{m}^{-3}\right]$ is the water density, $\mu\left[\mathrm{kg} \mathrm{m}^{-1} \mathrm{~s}^{-1}\right]$ is the water viscosity.

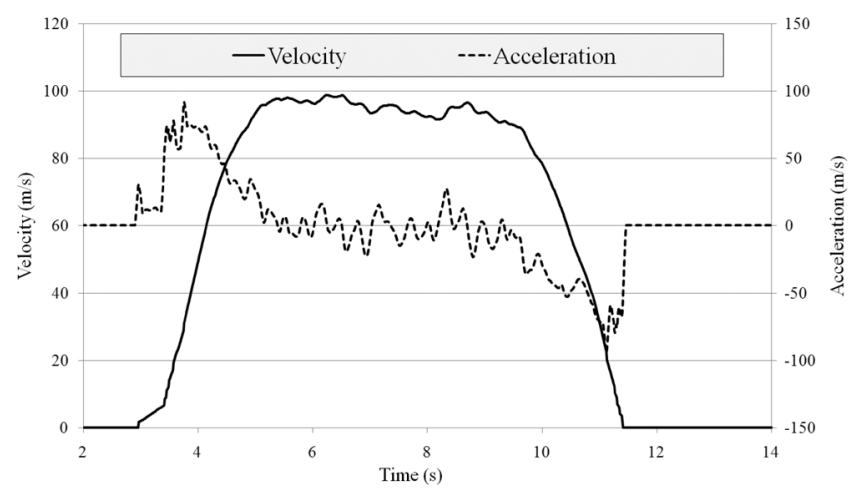

Figure 4: Velocity and acceleration of the rigid sheet.

Comparison between eqns (1) and (2) shows clearly that the pressure coefficient in hydrodynamics depends on new non-dimensional numbers, namely the Froude number, the acceleration and the non-dimensional depth. In fact, these terms appear in the derivation of eqn (1) but they appear to be negligible as 
long as the air domain may be considered infinite. This is the key point of the paper. So, the only mechanisms having an impact in aerodynamics flow cannot account for the whole drag pressure created in a free surface flow. Further mechanisms have to be identified (or have a significant impact). The question remains which ones? We aim hereafter at identifying these fundamental hydrodynamic mechanisms. Such identification is indeed a prerequisite to develop an accurate numerical algorithm for the simulation of the flow induced by a vertical moving body. Indeed, all phenomena involved have to be described in sufficient details by the set of equations and the boundary conditions.

\section{Results and interpretation}

\subsection{Constant velocity trial - experimental results}

First trial is a vertical translation at a velocity of $95 \mathrm{~mm} / \mathrm{s}$. Figure 4 shows the velocity and acceleration of the sheet during the translation. Three distinct stages of the motion are observed:

- Stage 1 - Acceleration from $0 \mathrm{~mm} / \mathrm{s}$ up to $95 \mathrm{~mm} / \mathrm{s}$.

- Stage 2 - Translation at constant velocity.

- $\quad$ Stage 3 - Deceleration down to $0 \mathrm{~mm} / \mathrm{s}$.

The evolution of the free surface height as a function of the water depth corresponding to the rigid sheet is shown in Figure 5a. The two curves show a sudden but small increase of the free surface level during the initial acceleration and a sharp decrease during the final deceleration. In-between these peaks, the free surface levels off. It suggests that the positive and negative acceleration stages are responsible for a wider extent of the free surface variation than the translation stage. What is more, it seems the impact of the translation varies with the water depth. Figure $5 \mathrm{~b}$ and $\mathrm{c}$ present the temporal evolution of the drag pressure (as defined in section 2.4) all along the vertical translation of the rigid sheet. A qualitative analyse of the curve related to the upper face shows a sudden increase during the acceleration stage up to a positive and nearly constant value during the translation stage. The deceleration stage comes with a sharp decrease of the drag pressure. For the lower face, the acceleration stage is characterized by a negative peak of pressure. The translation stage appears to be very changeable with a small bias toward negative pressure. Comparison between the values at the central and at the lateral gauges highlights a similarity of behaviour. Still, consistent differences are noted, especially as the panel reaches the free surface. The charts indicate indeed that drag pressures grow stronger at central gauges. In Figure $5 \mathrm{~b}$ and $\mathrm{c}$, pressure returns progressively to zero after a few minutes. Charts in this paper do not show this stabilization phase of the free surface, as it contains no relevant information.

\subsection{Controlled power trial - experimental results}

Second trial is a vertical translation with a fixed counterweight of $20 \mathrm{~kg}$. So, the energy provided to the panel is controlled. Figure 6 gives the velocity and 


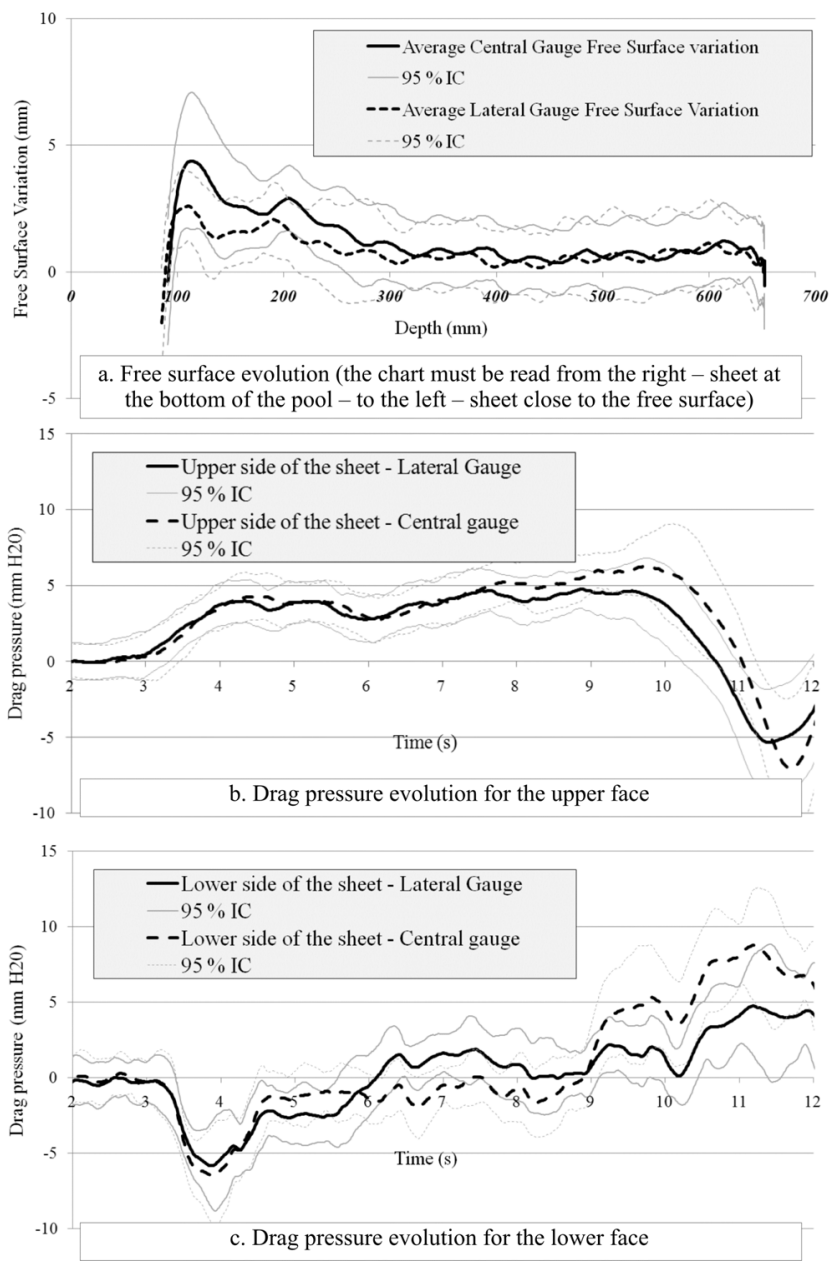

Figure 5: $\quad$ Experimental results for the constant velocity trial.

acceleration of the sheet along the translation. Two distinct stages of the motion are observed:

- Stage 1 - Acceleration from $0 \mathrm{~mm} / \mathrm{s}$ to the maximum velocity of $160 \mathrm{~mm} / \mathrm{s}$.

- $\quad$ Stage 2 - Deceleration down to zero velocity.

The evolution of the free surface level as a function of the water depth corresponding to the rigid sheet is shown in Figure 7a. The two curves show a sudden but small increase of the free surface level during the initial acceleration and a sharp decrease during the final deceleration. In-between these peaks, the free surface has been rising steadily. Figure $7 \mathrm{~b}$ and $\mathrm{c}$ illustrate the temporal evolution of the drag pressure all along the vertical translation of the panel. A qualitative analysis of the curve related to the upper face shows a small increase during the acceleration, followed by a continuous rising as long as the speed is 


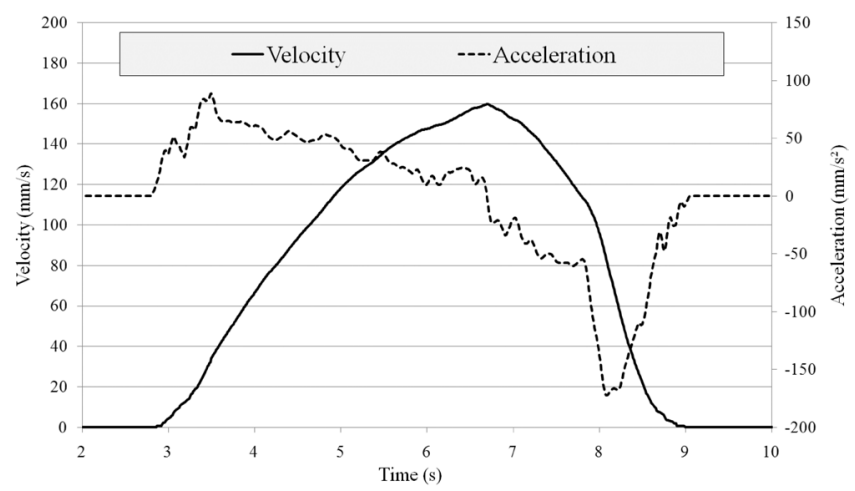

Figure 6: Velocity and acceleration of the rigid sheet.

increasing. The deceleration stage comes with a sharp decrease of the drag pressure. For the lower face, the acceleration stage is characterized by a negative peak of pressure. The deceleration stage comes with a positive peak of pressure. In-between, the pressure has been increasing steadily.

\subsection{Interpretation}

If we compare pressure measured and pressure expected according to eqn (1), it is clear that further contributions have to be identified in addition to the three mechanisms outlined in paragraph 3 . Based on the theoretical investigations and experimental results given above, authors propose three additional components. First, part of the hydrodynamic load provided to the panel by the operation device is consumed to prevail fluid inertia. For a sheet moving at constant velocity, Bernoulli equation can be written

$$
\frac{d}{d s}\left(\frac{U^{2}}{2 g}+\frac{p}{\rho g}+z\right)=-\frac{1}{g} \frac{\partial U}{\partial t}
$$

where $\mathrm{U}\left[\mathrm{ms}^{-1}\right]$ is the fluid velocity, $\mathrm{z}[\mathrm{m}]$ is the height and $\mathrm{s}[\mathrm{m}]$ is a curvilinear abscissa in the direction of the flow. The term of the right side of the equation (time derivative) accounts for the fluid inertia. As long as the fluid velocity is increasing, this term reinforces the positive pressure on the upper face of the panel and increases the negative pressure on the lower face. Kerger [5] rigorously demonstrated that the inertia term is inversely proportional to the square of the panel depth (which is defined as the distance between the free surface and the upper face of the panel). Second, the water and the panel exchange mass momentum during the acceleration and deceleration of the rigid sheet. Third, a significant part of the hydrodynamic load provided to the panel is consumed by propagation of free surface waves. The free surface waves are created to equilibrate the pressure increase induced to prevail the fluid inertia. Indeed, according to eqn (3), the fluid inertia induces a pressure increase given by eqn (4) below the free surface: 


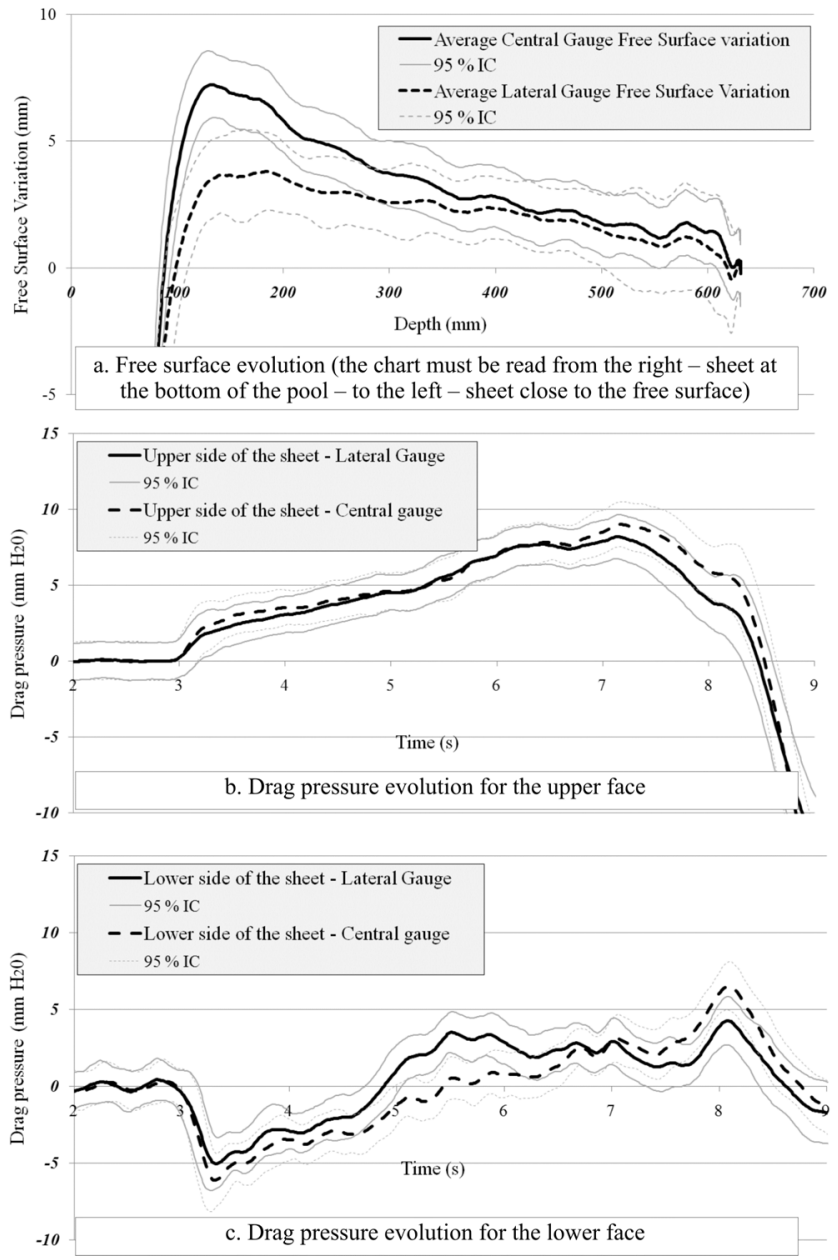

Figure 7: Experimental results for the controlled power trial.

$$
\Delta p=-\frac{1}{g} \frac{\partial U}{\partial t}
$$

The free surface level is however fixed by the local equilibrium of pressure $\mathrm{p}_{\text {atmospheric }}+\mathrm{p}_{\mathrm{s}}=\mathrm{p}_{\text {water }}$ where $\mathrm{p}_{\mathrm{s}}$ is the contribution of the surface stresses. Then, an increase of the water level is needed to equilibrate this rising of pressure.

\section{Conclusion}

As explained in the introduction, the authors propose in this paper a contribution to the understanding of experimental free surface flow induced by bodies moving upward. The study includes an overall analysis of the pressure field induced on 
the vertical uplifting of a rigid sheet. Six mechanisms identified and described in paragraph 4.3 give some basis to understand and predict the fluid behaviour and the fluid-structure interaction. In particular, evidence exposed before suggest the free surface has an active role in the creation of the pressure field. The fourth mechanism, involving the fluid inertia, becomes insignificant far away from the free surface. The same conclusion applies to the sixth mechanism, involving free surface waves. In conclusion, further research is needed to achieve a whole understanding of the behaviour of a body in motion in a free surface fluid. Similar experimental analysis for complex motion and/or complex body geometry allow to complete the experimental data, first step to a comprehensive fitting of pressure coefficient prescribed to a secure design of submerged moving structures.

\section{References}

[1] Canor A., Analyse expérimentale des effets induits par le mouvement de redressement d'une porte basculante, Master thesis, University of Liège, 2006.

[2] Wickert, G. \& Schmausser G., Stahlwasserbau, ed. Springer-Verlag. BerlinHeidelberg-New York, 1971.

[3] Dewals B.J., Erpicum S., Archambeau P., Detrembleur S. \& Pirotton M., Depth-Integrated Flow Modelling Taking into Account Bottom Curvature, Journal of Hydraulic Research, 44(6), 787-795, 2006.

[4] Detrembleur S., Archambeau P., Erpicum S., Dewals B.J. \& Pirotton M., An Explicit Projection Method for Solving Incompressible Navier-Stokes Equations, Proceedings of the International Junior Researcher and Engineer Workshop on Hydraulic Structures IJREWHS'08, PISA, 2008.

[5] Kerger, F., Analyse expérimentale des effets induits par le mouvement de redressement d'une porte basculante, Master thesis, University of Liège, 2007.

[6] Candel S., Mécanique des fluides, ed. Dunod. Vol. 1-2., Paris, 1990. 